\title{
Two-photon absorption related properties of functionalized BODIPY dyes in the infrared range up to telecommunication wavelengths $* *$
}

By Pierre-Antoine Bouit, Kenji Kamada, Patrick Feneyrou, Gérard Berginc, Lö̈c Toupet, Olivier Maury* and Chantal Andraud*.

[*] Dr. C. Andraud, P.-A. Bouit, Dr. O. Maury

Université de Lyon, ICL, CNRS - Ecole Normale Supérieure de Lyon, 46 allée d'Italie, 69007 Lyon (France)

E-mail: chantal.andraud@ens-lyon.fr

Dr. K. Kamada

Photonics Research Institute, AIST

AIST Kansai Center, 1-8-31 Midorigaoka, Ikeda, Osaka 563-8577, (Japan)

Dr. P. Feneyrou

Thales Research \& Technology France

Route Départementale 128, 91767 Palaiseau cedex, (France)

Dr. G. Berginc

Thales Optronique SA

BP 55, rue Guynemer, 78283 Guyancourt, (France)

Dr. L. Toupet

Groupe de la Matière Condensée et Matériaux, UMR 6626 CNRS-Université de Rennes 1 Campus de Beaulieu, 35042 Rennes cedex, (France)

[**] The authors thank the Direction Générale de l'Armement for a grant to PAB ((Supporting Information is available online from Wiley InterScience or from the author)).

Keywords: BODIPY, Two-photon absorption, Near infrared, Telecommunication wavelengths, nonlinear transmission

Two-photon absorption (TPA) is a resonant third-order nonlinear optical (NLO) process, in which an electron is promoted from its ground state to an excited state by simultaneous absorption of two photons of half-energy in a focused intense light beam like that generated by a laser source. This phenomenon, theoretically predicted in 1931 by Göppert-Mayer,${ }^{[1]}$ has been experimentally evidenced in 1961 in a TPA-induced fluorescence of $\mathrm{Eu}^{2+}$ doped $\mathrm{CaF}_{2}$ crystals under ruby laser irradiation. ${ }^{[2]}$ Since then, the intrinsic advantages of TPA excitation i.e. long wavelength and confocal three-dimensionally (3D) resolved absorption, led to 
numerous applications in the fields of material sciences and biology following the availability of tunable laser sources. In particular the development of fs-Ti-sapphire laser triggers numerous studies in the $700-1000 \mathrm{~nm}$ spectral range. For instance TPA was successfully involved in the fabrication of microstructures ${ }^{[3-4]}$ or high density optical memories ${ }^{[5]}$ with a 3D sub-micrometer resolution, in signal processing or for the design of optical limiting devices devoted to the protection of sensors against laser damages. ${ }^{[6-7]}$ In life sciences, the long wavelength TPA excitation is located in the biological transparency spectral range (800$1000 \mathrm{~nm}$ ), and therefore is able to penetrate more deeply biological tissues. Combined with the above mentioned spatial resolution, TPA found exciting applications for bio-imaging using nonlinear microscopy ${ }^{[8]}$ but also for drug delivery or dynamic phototherapy. ${ }^{[9]}$ All these applications encouraged the scientific community to design a large variety of chromophores with optimised two-photon cross-section $\left(\sigma_{2}\right)$ in this $700-1000 \mathrm{~nm}$ spectral range, e.g. organic dyes, organometallic or coordination complexes, polymers, dendrimers or nanonoparticles and quantum-dots, compiled in a recent exhaustive review. ${ }^{[10]}$ However, the near infrared (NIR) spectral range has been less studied and in particular the telecommunication wavelengths spectral range $(1.3-1.55 \mu \mathrm{m})$ where applications in signal processing (optical power stabilization, pulse suppression, optical limiting) are very attractive. Since 2005 , under the impulsion of the groups of Marder-Van Stryland and Osuka-Kim, there is a great interest for the design of chromophores exhibiting strong TPA properties in the NIR. Maximal TPA cross-section of ca. $1500 \mathrm{GM}$ at $1.44 \mu \mathrm{m}$ and $1600 \mathrm{GM}$ at $1.3 \mu \mathrm{m}$ have been reported for dipolar and quadrupolar squarine type chromophores respectively, ${ }^{[11-12]}$ whereas nickel(bisdithiolene) complexes ${ }^{[13]}$ and singlet diradical systems ${ }^{[14]}$ exhibit significant $\sigma_{2}$ over the entire telecommunication range. On the other hand, numerous fused-, extended- or organized-porphyrin complexes have been reported with very large TPA cross-section ranging from $10^{3}$ up to $10^{4} \mathrm{GM} \cdot{ }^{[15-19]}$ Finally we reported the TPA properties of cyanine-type chromophores $\left(\sigma_{2}\right.$ of $750 \mathrm{GM}$ at $1450 \mathrm{~nm}$ ) that present enough solubility to carry out 
additional nonlinear transmittance experiments at telecommunication wavelengths. ${ }^{[20]}$ Herein we report the synthesis, X-rays structures of aza boron-dipyrromethane (BODIPY) NIR dyes functionalized in the $\alpha$ position by donor- $\pi$-conjugated systems and their TPA and nonlinear transmittance properties in the 1.2-1.6 $\mu \mathrm{m}$ spectral range.

It is worth noting that BODIPY is a versatile class of chromophores ${ }^{[21-22]}$ largely studied for its exceptionnal luminescence properties and used as biomolecular labels, sensors (pH, NO, $\mathrm{Hg}^{2+}$, toxin...) or photonic therapeutic agent. ${ }^{[21-26]}$ Great efforts have been recently devoted to shift their spectroscopic properties to the NIR spectral range by (i) introduction of electron donating moieties, ${ }^{[27]}$ (ii) rigidification of the structure ${ }^{[28]}$ or (iii) replacement of the mesocarbon by a nitrogen atom (the "aza"-BODIPY). ${ }^{[29-31]}$ But surprisingly, whereas water soluble conventional BODIPY dyes featuring low two-photon cross-section were used for two-photon imaging microscopy, ${ }^{[32-33]}$ these compounds were not fully optimized for TPA applications. To the best of our knowledge, only multichromophoric dyes containing two or three BODIPY units and exhibiting rather modest $\sigma_{2}$ values $(75 \mathrm{GM}$ at $990 \mathrm{~nm})$ in the visible were described in the literature. ${ }^{[34]}$

The synthesis of the target chromophores $\mathbf{B}^{\mathbf{1 - 2}}$ (Scheme 1), featuring extended $\pi$-conjugated skeleton involves as key step a copper free Sonogashira cross-coupling using $\mathrm{Pd}\left(\mathrm{PPh}_{3}\right)_{4}$ as catalyst between bromo-functionalized aza-dipyrromethene ${ }^{[35]}$ and various donorphenylenethynyl derivative $\left(\mathrm{D}=\mathrm{OHex}, \mathrm{D}=\mathrm{NHex}_{2}\right.$ ) followed by classical complexation with boron trifluoride etherate in basic media (Scheme 1). The intermediate synthon 3 and the corresponding complex $\mathbf{B}^{\mathbf{0}}$ are prepared using the procedure described by the O'Shea's group. ${ }^{[36]}$ Synthetic details and complete characterization are compiled in supporting information. It is important to note that the synthesis of $\mathbf{B}^{\mathbf{0 - 2}}$ was carried out at a gram scale and $\mathbf{B}^{2}$ is enough soluble in chlorinated solvent to envisage further nonlinear transmittance experiments. ${ }^{[20]}$ 

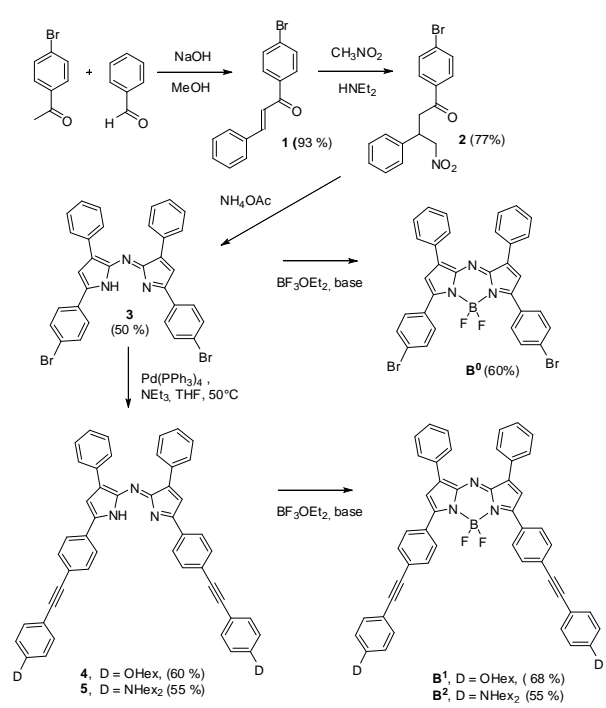

Scheme 1. Synthesis of chromophores.

Crystals of $\mathbf{B}^{\mathbf{0}}$ suitable for X-ray diffraction analysis were obtained by slow diffusion of diethylether into a dichloromethane solution. As already observed for other aza-BODIPY dyes, ${ }^{[31,35,37-38]}$ the structure (Figure $\mathrm{S} 1$ in supporting informations) shows three very planar central fused rings and four twisted peripherical phenyl groups (twist angle of $12-43^{\circ}$ ); the boron atom lies in an almost perfect tetrahedral environment and the $\mathrm{C}-\mathrm{N}$ bond lengths between bridging nitrogen N2 and the two pyrroles C4 and C5 are identical (1.322(3) and $1.326(3) \AA$, respectively), showing the conjugated nature of the chromophore.

All the linear and nonlinear properties were studied in dichloromethane solution and are summarized in Table 1. Absorption spectrum (Fig.1) of $\mathbf{B}^{\mathbf{0}}$ is characteristic of aza-BODIPY with an intense sharp transition $\left(\omega_{1 / 2}=1270 \mathrm{~cm}^{-1}\right)$ exhibiting a shoulder at higher energy. The extended $\pi$-conjugated $\mathbf{B}^{\mathbf{1 , 2}}$ compounds present transitions whose red shift increases with the strength of the donor group ( $\Delta \lambda=37$ and $87 \mathrm{~nm}$ for $\mathbf{B}^{\mathbf{1}}$ and $\mathbf{B}^{\mathbf{2}}$, respectively). It is worth noting that this shift is accompanied by a significant broadening of the absorption band $\left(\omega_{1 / 2}=\right.$ $2850 \mathrm{~cm}^{-1}$ for the amino derivative). 


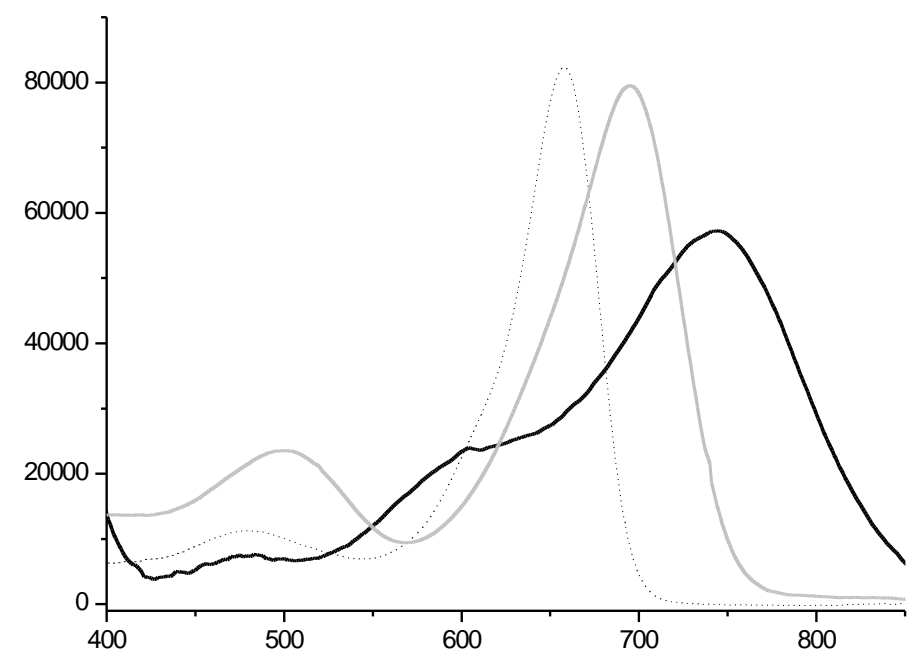

Figure 1. Absorption spectra of $\mathbf{B}^{\mathbf{0}}$ (dashed), $\mathbf{B}^{\mathbf{1}}$ (gray) and $\mathbf{B}^{\mathbf{2}}$ (bold).

Open aperture Z-scan measurements were performed in the 1200-1600 nm spectral range using a fs-optical parametric amplifier pumped by a Ti:sapphire source. At such short pulse duration (130 fs), only TPA process is considered and the propagation equation can be simplified as:

$d I / d z=-\alpha_{2} I^{2}$

where $I$ represents the light intensity, $z$ the propagation axis and $\alpha_{2}$ the TPA coefficient. As described previously, ${ }^{[20]}$ the $\alpha_{2}$ coefficient can be determined from the molecular two-photon cross-section $\sigma_{2}$ by:

$$
\sigma_{2}=h v \alpha_{2} / N
$$

Carrying these fs-Z-scan experiments at various wavelengths allowed the determination of the TPA spectra of $\mathbf{B}^{\mathbf{0 , 1}, \mathbf{2}}$ over the entire 1200-1600 nm spectral range (Fig.2). As expected for non-centrosymmetric compounds, each TPA spectrum matches the wavelength doubled linear one, indicating that the same transition is one- and two-photon allowed. At these wavelengths, 
the unfunctionalized $\mathbf{B}^{\mathbf{0}}$ chromophore does not present any TPA response in the contrary of the donor- $\pi$-conjugated substituted systems $\mathbf{B}^{\mathbf{1 , 2}}$ (Table 1). Whereas $\mathbf{B}^{\mathbf{1}}$ presents a rather small NLO activity, $\mathbf{B}^{2}$ shows significant $\sigma_{2}$ values (around $600 \mathrm{GM}$ ) over the broad 1300$1450 \mathrm{~nm}$ spectral range. Furthermore, at lower wavelength, a significant increase is observed with a maximal two-photon cross-section $\sigma_{2}^{\max }$ of $1070 \mathrm{GM}$ at $1220 \mathrm{~nm}$. This value is close to that of cyanine compounds ${ }^{[20]}$ and in the same order of magnitude than that of dipolar or quadrupolar derivatives. ${ }^{[11,12]}$

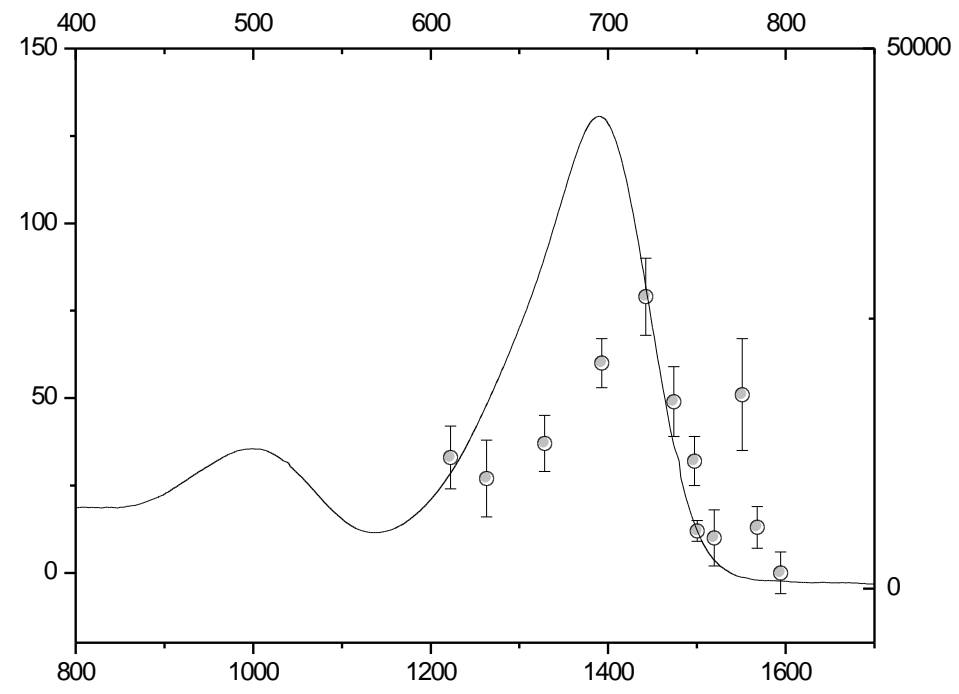




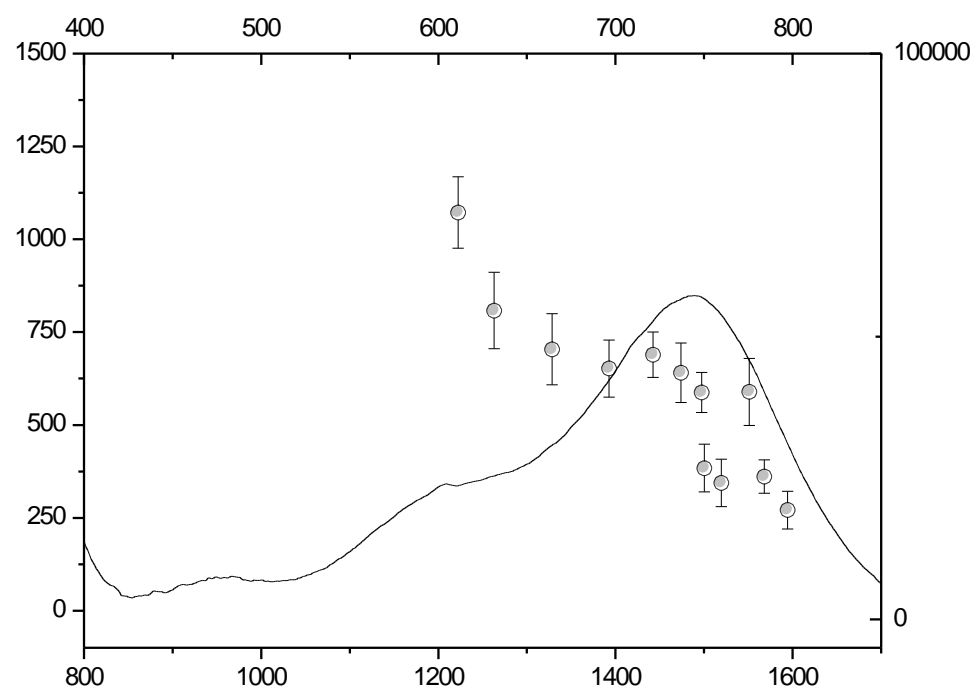

Figure 2. TPA spectra measured by fs Z-Scan method (filled circle) and linear absorption spectra in upper abscissa (-) for $\mathbf{B}^{\mathbf{1}}$ (up) and $\mathbf{B}^{\mathbf{2}}$ (bottom).

In addition to the high solubility, exceptional thermal stability $\left(\mathrm{T}_{\mathrm{d} 10}\right.$ about $\left.360{ }^{\circ} \mathrm{C}\right)$ and the large scale availability of $\mathbf{B}^{2}$ allowed to perform nonlinear transmittance experiments in the telecommunication spectral range. Such experiments were carried out for $\mathbf{B}^{\mathbf{2}}$ at highly concentrated solution $\left(\mathrm{c}=0.1 \mathrm{Mol} \cdot \mathrm{L}^{-1}\right)$ using a ns-OPO pumped by a frequency tripled Nd:YAG laser source. ${ }^{[20]}$ Over the $1350-1500 \mathrm{~nm}$ range, the nonlinear transmittance curves vs. the laser fluence present characteristic nonlinear transmission properties; this behavior is illustrated at $1350 \mathrm{~nm}$ in Figure 3: a good transmission is observed at low energy $(\mathrm{T}>0.9)$, whereas beyond the nonlinear transmission threshold observed for a fluence of $0.7 \mathrm{~J} . \mathrm{cm}^{-2}$, the laser beam intensity is strongly attenuated up to $60 \%$ at a fluence of $7 \mathrm{~J} . c m^{-2}$. Due to the longer pulse duration ( $7 \mathrm{~ns}$ ), the simulation of the experimental curves based only on a TPA process failed (blue line, Figure 3) and a higher order effect related to a three-photon absorption, such as a TPA induced excited state absorption (ESA) was taken into account. 
Consequently, as already observed in the NIR for cyanine dyes ${ }^{[20]}$ or in the visible, ${ }^{[39-40]}$ the propagation equation (1) becomes

$d I / d z=-\alpha_{2} I^{2}-\alpha_{3} I^{3}$

where $\alpha_{3}$ is the three-photon absorption coefficient. Numerical resolution of (3), for temporally and spatially gaussian incident pulses and using the TPA coefficient previously measured by fs-Z-scan, allowed the determination of a $\alpha_{3}$ coefficient of $620 \mathrm{~cm}^{3} . \mathrm{GW}^{-2}$ at 1350 nm (Table 1). This value corresponds to a two-fold increase compared to that of cyanine dyes measured in the same condition of concentration $\left(230 \mathrm{~cm}^{3} \cdot \mathrm{GW}^{-2}\right.$ at $\left.1520 \mathrm{~nm}\right) .{ }^{[20]}$ This enhancement, which cannot be interpreted only by a stronger TPA efficiency, could be explained by a strong ESA contribution. This is illustrated in Figure S1 in supporting informations by the comparison of the spectral distribution of $\alpha_{2}$ and $\alpha_{3}$ between 1350 and $1500 \mathrm{~nm}: \alpha_{3}$ decreases from 600 to $300 \mathrm{~cm}^{3} . \mathrm{GW}^{-2}$, while $\alpha_{2}$ remains nearly constant. 


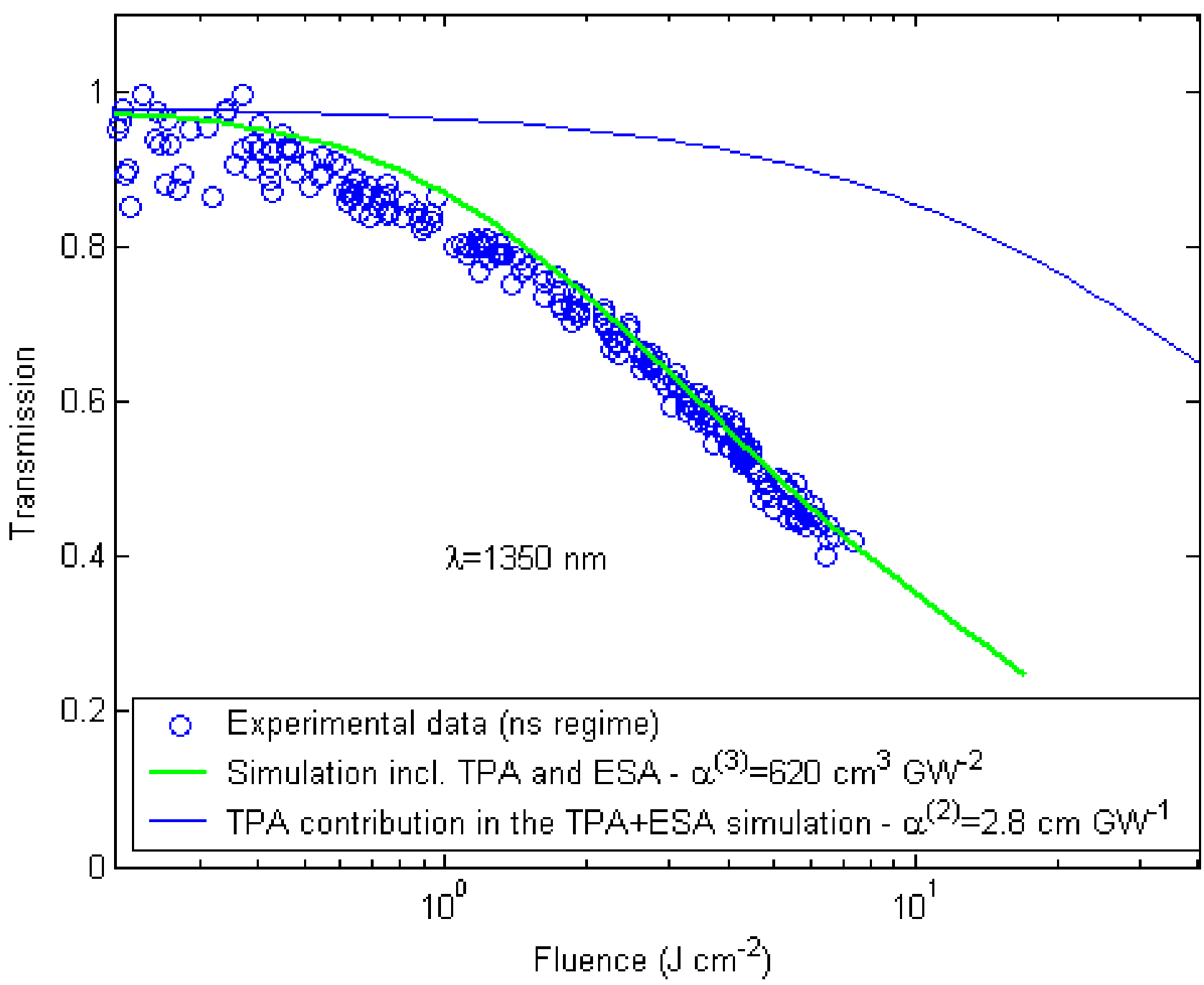

Figure 3. Transmittance variations with the laser fluence in a DCM solution of $\mathbf{B}^{\mathbf{2}}$ at a concentration of 106 g.L $\mathrm{L}^{-1}$; experimental data points (blue circle), theoretical simulation using complete model including TPA and ESA contribution (green), including only the TPA contribution within this latter hypothesis.

In conclusion, this study clearly underlines the high potentialities of functionalized azaBODIPY dyes for TPA related applications in the telecommunication spectral range and in particular for nonlinear transmission. The versatile nature of this class of chromophores opens the way for a fine chemical engineering in order to optimize the TPA properties in the NIR by 
additional functionalization of the $\beta$ position or by rigidification of the lateral $\pi$-skeleton for example.

\section{Experimental}

[CCDC- 668638 contains the supplementary crystallographic data for this paper. These data can be obtained free of charge from The Cambridge Crystallographic Data Centre via www.ccdc.cam.ac.uk/data_request/cif.]

[Further details of the crystal structure investigation(s) may be obtained from the Fachinformationszentrum Karlsruhe, 76344 Eggenstein-Leopoldshafen (Germany), on quoting the depository number CSD-...]

[1] M. Göppert-Mayer, Naturwissenschaften, 17, 932.

[2] W. Kaiser, C. G. B. Garret Phys. Rev. Lett., 1961, 7, 229.

[3] I. Wang, M. Bouriau, P.L. Baldeck, C. Martineau, C. Andraud Opt. Lett. 2002, 27, 1348.

[4] K.-S. Lee, D.-Y. Yang, S. H. Park, R. H. Kim Polym. Adv. Technol. 2006, 17, 72.

[5] S. Kawata, H.-B. Sun, T. Tanaka, K. Takada, Nature, 2001, 412, 697.

[6] C.W. Spangler, J. Mater. Chem., 1999, 9, 2013.

[7] Y. Morel, A. Irimia, P. Najechalski, O. Stephan, P. L. Baldeck, C. Andraud J. Chem.Phys. 2001, 114, 5391

[8] A. Picot, A. D’Aléo, P. L. Baldeck, A. Grishine, A. Duperray, C. Andraud, O. Maury J. Am. Chem. Soc., 2008, 130, 1532. 
[9] W.R. Ziepfel, R.M. Williams, W.W. Webb, Nature Biotech., 2003, 21, 1369.

[10] G.S. He, L.-S. Tan, Q. Zheng, P.N. Prasad, Chem. Rev., 2008, 108, 1245.

[11] L. Beverina, J. Fu, A. Leclercq, E. Zojer, P. Pacher, S. Barlow, E.W. Van Stryland, D. J. Hagan, J.-L. Brédas, S.R. Marder, J. Am. Chem. Soc. 2005, 127, 7282.

[12] S. J. Chung, S. Zheng, T. Odani, L. Beverina, J. Fu, L.A. Padilha, A. Biesso, J.M. Hales, X. Zhan, K. Schmidt, A. Ye, E. Zojer, S. Barlow, D.J. Hagan, E.W. Van Stryland, Y. Yi, Z. Shuai, G. A. Pagani, J.-L. Brédas, J.W. Perry, S. R. Marder, J. Am. Chem. Soc. 2006, $128,14444$.

[13] J.-Y. Cho, S. Barlow, S.R. Marder, J. Fu, L.A. Padilha, E.W. Van Stryland, D.J. Hagan, M. Bishop, Opt. Lett. 2007, 32, 671.

[14] K. Kamada, K. Ohta, T. Kubo, A.Shimizu, Y. Morita, K. Nakasuji, R. Kishi, S. Ohta, S. Furukawa, H. Takahashi, M. Nakano Angew. Chem. Int. Ed. 2007, 46, 3544.

[15] Y. Inokuma, N. Ono, H. Uno, D.Y. Kim, S.B. Noh, D. Kim, A. Osuka, Chem. Commun. 2005,3782 .

[16] K. Kurotobi, K.S. Kim, S.B. Noh, D. Kim, A. Osuka, Angew. Chem. Int. Ed. 2006, 45, 3944.

[17] S. Mori, K.S. Kim, Z.S. Yoon, S.B. Noh, D. Kim, A. Osuka, J. Am. Chem. Soc. 2007, $129,11344$.

[18] Y. Tanaka, S. Saito, S. Mori, N. Aratani, H. Shinokubo, N. Shibata, Y. Higuchi, Z.S. Yoon, K.S. Kim, S.B. Noh, J.K. Park, D. Kim, A. Osuka, Angew. Chem. Int. Ed. 2008, 47,681 .

[19] M. Drobizhev, Y. Stepanenko, A. Rebane, C. J. Wilson, T. E. O. Screen, H. L. Anderson, J. Am. Chem. Soc. 2006, 128, 12432.

[20] P.-A. Bouit, G. Wetzel, G. Berginc, L. Toupet, P. Feneyrou, Y. Bretonnière, O. Maury, C. Andraud Chem. Mater. 2007, 19, 5325 .

[21] A. Loudet, K. Burgess, Chem. Rev. 2007, 107, 4891. 
[22] G. Ulrich, R. Ziessel, A. Harriman, Angew. Chem. Int. Ed., 2007, 46, 2.

[23] Y. Gabe, Y. Urano, K. Kikuchi, H. Kojima, T. Nagano, J. Am. Chem. Soc., 2004, 126, 3357.

[24] R.E. Gawley, H. Mao, M.M. haque, J.B. Thorne, J.S. Pharr, J. Org. Chem., 2007, 72, 2187.

[25] A. Coskun, M.D. Yilmaz, E.U. Akkaya, Org. Lett., 2007, 9, 607.

[26] S.O. McDonnell, M.J. Hall, L.T. Allen, A. Byrne, W.M. Gallagher, D.F. O’Shea, J. Am. Chem. Soc., 2005, 127, 16360

[27] Y.-H. Yu, A. B. Descalzo, Z. Shen, H. Röhr, Q. Liu, Y.-W. Wang, M. Spieles, Y.-Z. Li, K. Rurack, X.-Z. You Chem. Asian J., 2006, 1, 176-187.

[28] W. Zhao, E.M. Carreira, . Angew. Chem .Int. Ed., 2005, 44, 1677.

[29] W. Zhao, E.M. Carreira, . Chem. Eur; J., 2006, 12, 7254.

[30] K. Umezawa, Y. Nakamura, H. Makino, D. Citterio, K. Suzuki, J. Am. Chem. Soc., 2008, $130,1550$.

[31] S.O. McDonnell, D.F. O’Shea, Org. Lett., 2006, 8, 3493.

[32] C. Nicoli, J. Baranski, S. Schlummer, J. Palomo, M. Lumbierres-Burgues, M. Kahms, J. Kuhlmann, S. Sanchez, E. Gratton, H. Waldmann, R. Winter, J. Am. Chem. Soc., 2006, $128,192$.

[33] F. Bestvater, E. Spiess, G. Stobrawa, M. Hacker, T. Feurer, T. Porwol, U. BerchnerPfannschmidt, C. Wostlaw, H. Acker, J. Microsc., 2002, 208, 108.

[34] L. Porres, O. Mongin, M. Blanchard-Desce Tetrahedron Lett., 2006, 47, 1913.

[35] Very recently, an iodo analogous of $\mathbf{B}^{\mathbf{0}}$ has been described: A. Loudet, R. Bandichhor, L. Wu, K. Burgess, Tetrahedron, 2008, 64, 3642.

[36] A. Gorman, J. Killoran, C. O’Shea, T. Kenna, W.M. Gallagher, D.F. O'Shea, J. Am. Chem. Soc., 2004, 126, 10619. 
[37] J. Killoran, L. Allen, J.F. Gallagher, W.M. Gallagher, D.F. O’Shea, Chem. Commun., 2002, 1862.

[38] J. Killoran, S.O. McDonnell, J.F. Gallagher, D.F. O’Shea, New. J. Chem., 2008, 32, 483.

[39] R. Anémian, Y. Morel, P.L. Baldeck, B. Paci, K. Kretsch, J.-M. Nunzi, C. Andraud, J. Mater. Chem., 2003, 13, 2157.

[40] C. Girardot, B. Cao, J.-C. Mulatier, P. L. Baldeck, J. Chauvin, D. Rhiel, J. A. Delaire, C. Andraud, G. Lemercier ChemPhysChem, 2008, 9, 1531. 